\title{
Chaotic Phenomena in Addiction Model for Digital Leisure
}

\section{Youngchul Bae}

Division of Electrical.Electronic Communication and Computer Engineering, Chonnan National University, Yeosu, Korea

\section{]jfis}

\begin{abstract}
Chaotic dynamics have been studied by many researchers in the fields of biology, physics, and engineering. Interest in chaos is also expanding to the social sciences such as politics, economics, and others, including the prediction of societal events. The concept of leisure has developed from a passive concept correlated with relaxation, entertainment, and ideology formation into a positive concept that assumes a more active role. As information and communications technology develops, digital leisure activity is expected to continue spreading. This expansion of digital leisure function correctly, as well as. Traditional leisure activity functions correctly more, whereas digital leisure activity is predicted to function incorrectly more often. In this paper, we propose a mathematical addiction model of digital leisure that deals with its dysfunctions such as addiction to digital leisure, including computer games, internet search, internet chatting, and social media. Herein, to solve addiction to digital leisure, we propose a model derived from a nicotine addiction.
\end{abstract}

Keywords: Chaotic dynamics, Nonlinear dynamics, Digital leisure, Addiction model, Leisure dysfunction

\section{Introduction}

Nonlinear dynamics, including chaotic dynamics, have been widely used in real-world applications such as biological systems [1], brain modeling [2], weather modeling [3], vibration modeling [4], mechanical and electrical engineering [5, 6], control and synchronization [7, 8], robotics [9, 10], and more [11-15]. Chaotic dynamics have also been studied by many re-

Received: Dec. 4, 2013

Revised : Dec. 23, 2013

Accepted: Dec. 24, 2013

Correspondence to: Youngchul Bae (ycbae@chonnam.ac.kr)

(cThe Korean Institute of Intelligent Systems

(c) This is an Open Access article distributed under the terms of the Creative Commons Attribution Non-Commercial License (http://creativecommons.org/licenses/ by-nc/3.0// which permits unrestricted noncommercial use, distribution, and reproduction in any medium, provided the original work is properly cited. searchers in the fields of biology, physics, and engineering. However, this research is not limited to the field of natural science only, but is now spreading to the social sciences such as politics, seconomics, and others, including the prediction of social societal events.

The concept of leisure has developed from a passive concept correlated with relaxation, entertainment, and ideology formation into a positive concept that assumes a more active role. As a consequence, attaining health and happiness has become an important aspect of life. This, in turn, has caused selectivity, flexibility, spontaneity, and self-decision to become essential elements for humanity to pursuite. Leisure can be divided into two main types: indoor and outdoor. Outdoor leisure includes activities such as running, hunting, and climbing. Recently, however, cyber or digital leisure, in which digital devices are used, has been established as one of the most popular leisure activities.

As information and communications technology develops, digital leisure activity is expected 
to continue spreading. This expansion of digital leisure can function correctly but also incorrectly. Traditional leisure activity functions correctly more often, whereas digital leisure activity is predicted to function incorrectly more often. In some developed countries, governments implement policies regarding leisure activity that includes protection from addiction to digital leisure, but in the Republic of Korea, the government assumes a passive role in establishing proper policies related to the measurement of leisure, despite its importance. Consequently, relevant government organizations should propose policies for preventing addiction to digital leisure.

Metin and Sengor [16] proposed a mathematical model nicotine addiction. However, a model for digital leisure has yet to be derived.

In this paper, we propose a mathematical addiction model of digital leisure that deals with dysfunctions such as addiction to digital leisure, including computer games, internet search, internet chatting, social media, and the like. As a step toward developing treatment for addiction to digital leisure, we propose a model for it derived from the nicotine addiction model [16]. Based on this derivation, we also propose a similarity between addiction to digital leisure and a route to chaos.

\section{Chaotic Dynamics}

Chaotic dynamics is defined as "an irregular oscillation governed by a relatively simple rule." In this paper, we consider the logistic map that was popularized by May [3] in 1976 to calculate population growth. The function of the logistic map is given by Eq. (1).

$$
X_{n+1}=\gamma X_{n}\left(1-X_{n}\right)
$$

From Eq. (1), we get a time series that appears to be a random signal, as shown in Figure 1.

We also obtain a phase portrait and iteration from Figure 1, as shown in Figure 2.

\section{Modeling of Addiction to Digital Leisure}

In this section, we establish a model of addiction to digital leisure, particularly addiction to games and the use of digital devices. Many neuroscientists have found that addiction involves an interaction between the limbic and cortical systems. Generally, all types of addiction such as nicotine addiction are known to result from a malfunction of the reward mechanism in the brain. Thus, if someone is addicted to a substance, he or she

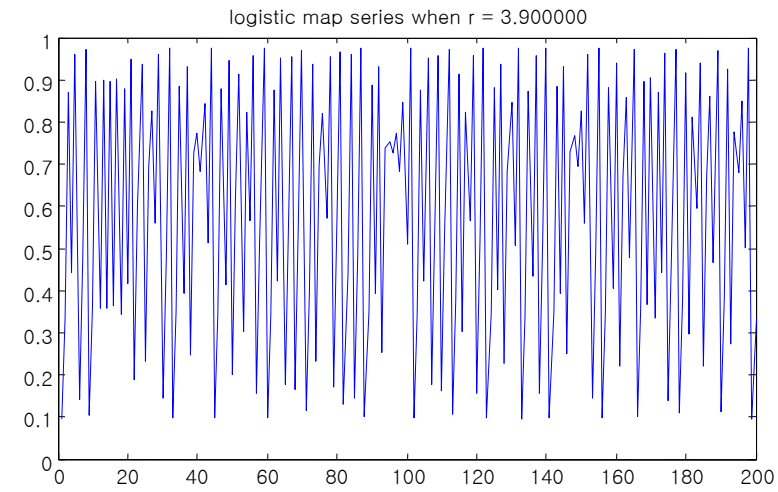

Figure 1. Time -series of logistic map.

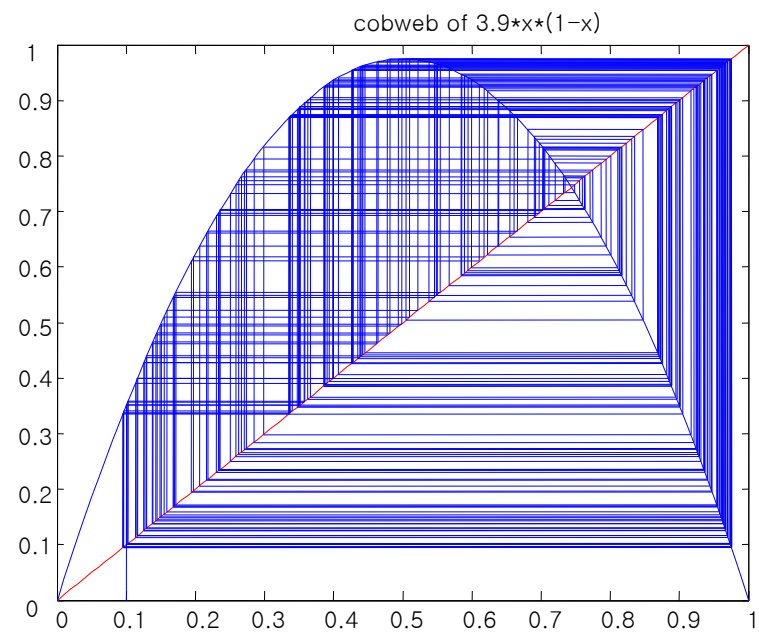

Figure 2. Phase portrait and iteration of logistic map.

craves that substance fanatically. For example, someone who is a nicotine-addict must obtain more nicotine continuously to satisfy the malfunctioning reward mechanism.

To construct a mathematical model for new addictions such as addiction to games and the usage of digital devices, we used the nicotine addiction Eq. (2) [16].

$u_{D A}(k+1)=u_{D A}(k)+m u_{D A}\left(-u_{D A}(k)+s_{D A}(r i, n i)\right)$

where $m u_{D A}$ is the learning rate in the DA subsystem, and $s_{D A}(r i, n i)$ is the activation function given as the sigmoid function in Eq. (3).

$$
s_{D A}(r i, t i)=0.5\left(1+\tanh \left(n i * r i-\theta_{D A}\right)\right)
$$

where, ni, ri, and $\theta_{D A}$ are the nicotine uptake signal, the reward 
signal initiated by nicotine, and the threshold setting the minimum tonic DA, respectively. We take $n i=0.1$ as a nicotine signal and $\theta_{D A}=0.1 * n i$.

From Eq. 22], the model of nicotine addiction, we can derive a model of addiction to digital leisure. To do this, we assume that the first and second terms of the right-hand side of Eq. (1) and the first and second terms of the right-hand side of Eq. (2), respectively, are equivalent.

Both Eqs. (1) and (2) are second-order systems, except for the sigmoid function in Eq. 22); therefore, we can write Eqs. (4) and (5).

$$
\begin{gathered}
\gamma X_{n}=u_{D A} \\
-\gamma X_{n}^{2}=-m u_{D A}^{2}
\end{gathered}
$$

Because the orders of Eqs. (1) and (2) are the same except for the sigmoid function in Eq. (2), we can use Eq. (1) instead of Eq. (2) as the addiction model of digital leisure.

Finally, we rewrite Eq. (1) as Eq. (6).

$$
X_{n+1}=a X_{n}\left(1-X_{n}\right)
$$

Now, we will review the situation of addiction to digital leisure with variation in the parameter $a$. In this paper, we assume that the parameter $\gamma$ is the degree of concentration, including time, period, repetition, and the like.

\subsection{Equilibrium Point (No Addiction Stage)}

When $a<3$, we reach an equilibrium point. For example when $a=0.5$, we have only a single equilibrium point at $x=0$. When $\mathrm{a}=2.0$, we have two equilibrium points at $x=0$ and $x=0.5$. When $\mathrm{a}=2.5$, we also have two equilibrium points at $x=0$ and $x=0.6$.

From Eq. (6), we get a graph to represent an equilibrium point with varying $\gamma$, shown in Figure 3 .

Typically, people begin using digital devices such as games and smart phones for their hobbies, simple curiosity, and/or work. This case seems to be displayed at the equilibrium point shown in the graph, because people who begin dealing with digital devices tend to lack familiarity with them. Consequently, those people will readily return to the previous state of not using the device. Thus, people who use a device out of simple curiosity or for a hobby are unlikely to reach the addiction stage.

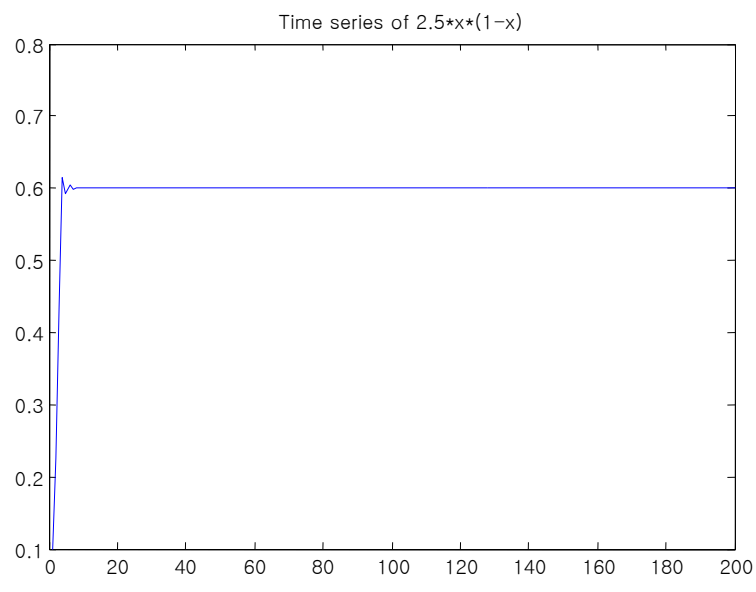

(a) Time series.

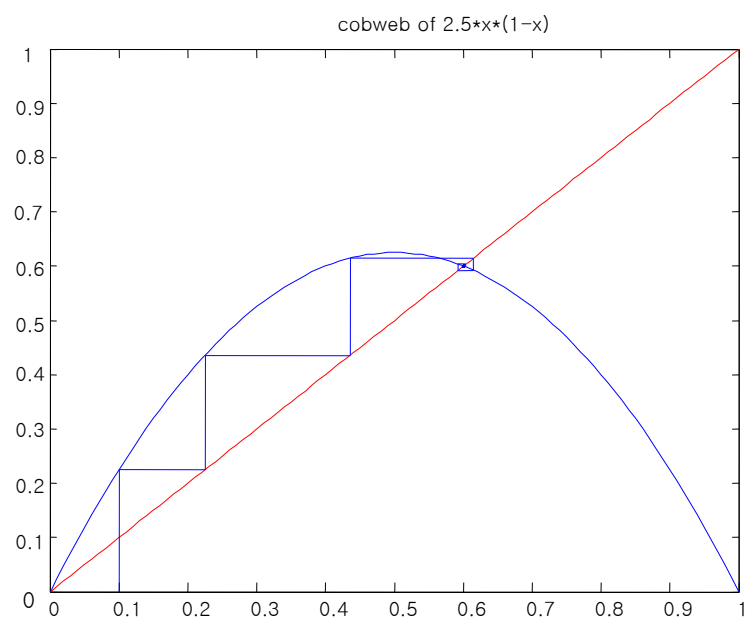

(b) Phase portrait and iteration with equilibrium point

Figure 3. Time series and phase portrait with equilibrium point of addiction to digital leisure when $\mathrm{a}=2$.

\subsection{Period-Doubling (Pre-Addiction Stage)}

When a increases, with $a<3.2$, the equilibrium point disappears and appears as a one- period motion at $a=3.2$. Figure 4 shows the time series, phase portrait, and iteration with equilibrium point when $\mathrm{a}=3.2$.

When $a$ has values in the ranges $3.2<a<3.449,3.449<$ $a<3.54409,3.54409<a<3.5644,3.5644<a<$ 3.56875, Eq. (6) displays 2-period, 4-period, 8-period, and 16-period orbits, respectively. Figures 5 and 6 shows the time series, phase portrait, and iteration with equilibrium point when $\mathrm{a}=3.4$ and $\mathrm{a}=3.5$, respectively. From these figures, we recognize that one is a 2-period and the other is a 4-period orbit.

The period-doubling case of Eq. (6) shows a pre-addiction 


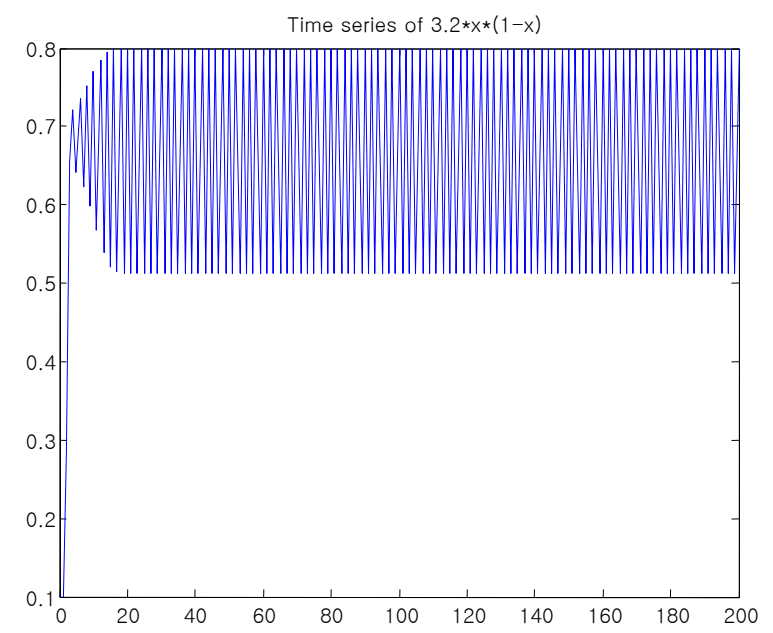

(a) Time series.

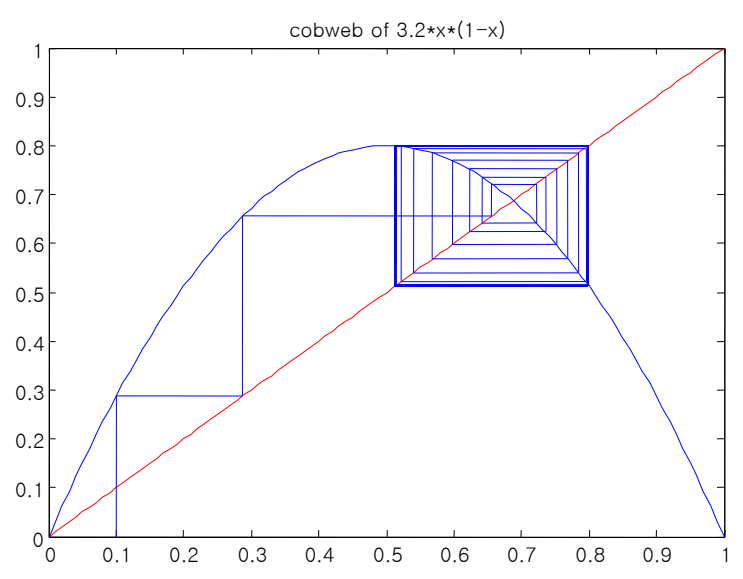

(b) Phase portrait and iteration with equilibrium point.

Figure 4. Time series and phase portrait with equilibrium point to addiction of digital leisure when $\mathrm{a}=3.2$.

stage. As people pay more attention to using digital devices, we may predict that the length of time they use those devices increases steadily. Because use is easier for people accustomed to digital devices as compared to beginners, they can focus more on using digital devices without having to deal with any discomfort from using them. However, if those people increase the time they use games or digital devices continuously, the 1-periodic pattern changes to 2- period and 4- period patterns. We call this pre-addiction stage in digital device usage periodic doubling, as in chaos dynamics.

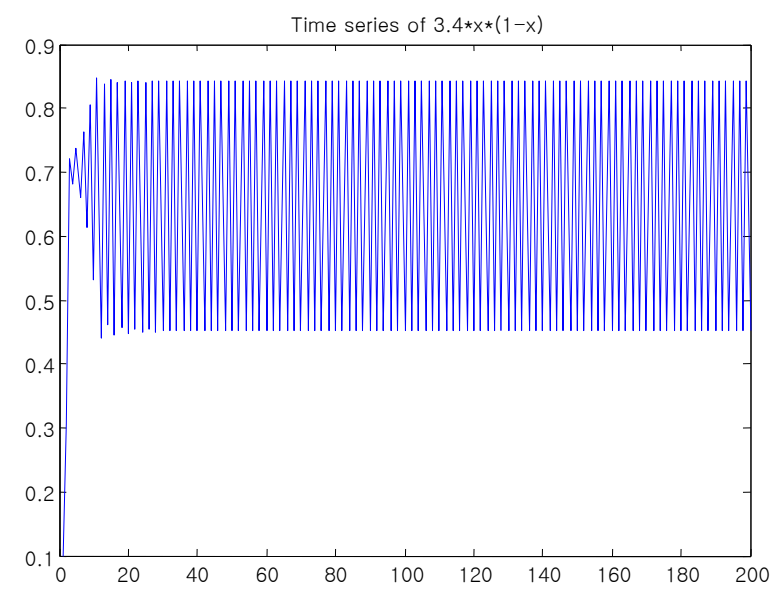

(a) Time series.

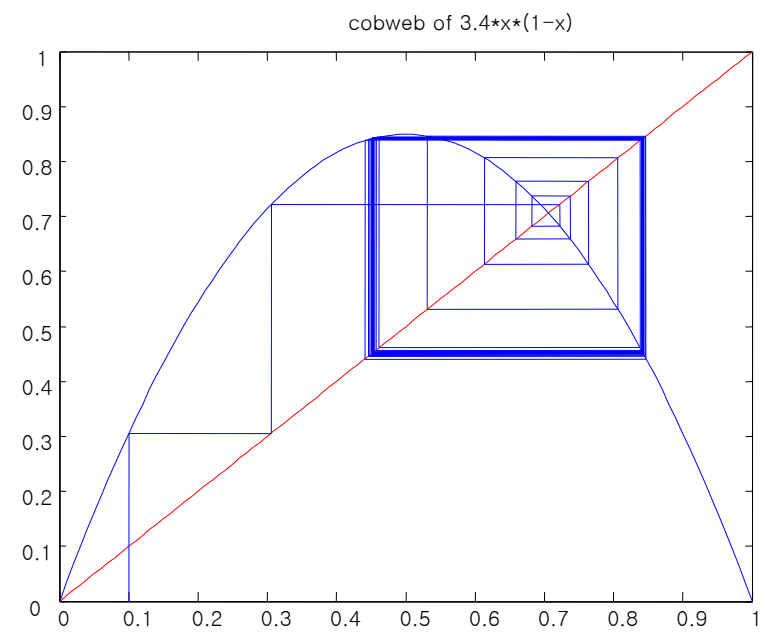

(b) Phase portrait and iteration with equilibrium point.

Figure 5. Time series and phase portrait with equilibrium point to addiction of digital leisure when $\mathrm{a}=3.4$.

\subsection{Quasi-Period and Chaos (Addiction Stage)}

If we increase a to $a>3.7$, then Eq. (1) reaches chaos. Figure 7 shows the time series, phase portrait, and iteration with the equilibrium point of Eq. (6).

If the users of digital devices increase their usage time, periodic doubling occurs as a consequence of our digital device model, and the periodic pattern changes suddenly from periodic to chaotic. In our view, this represents addiction to games or digital devices, similar to a chaotic situation in chaotic dynamics. 


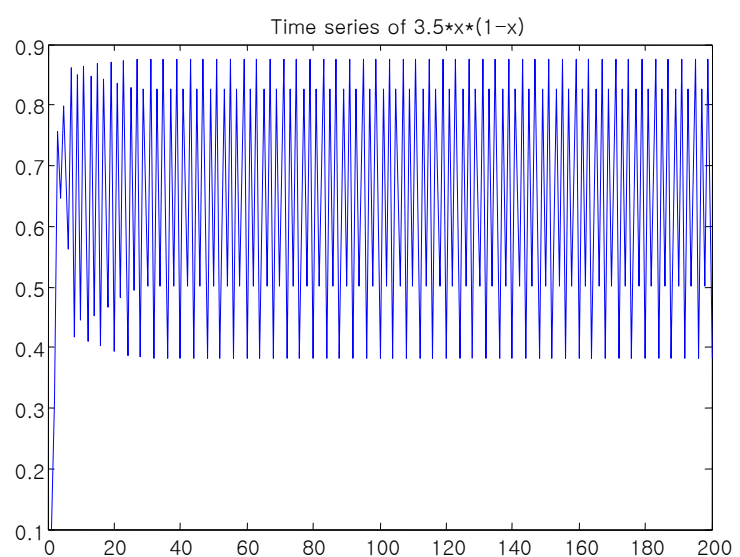

(a) Time series.

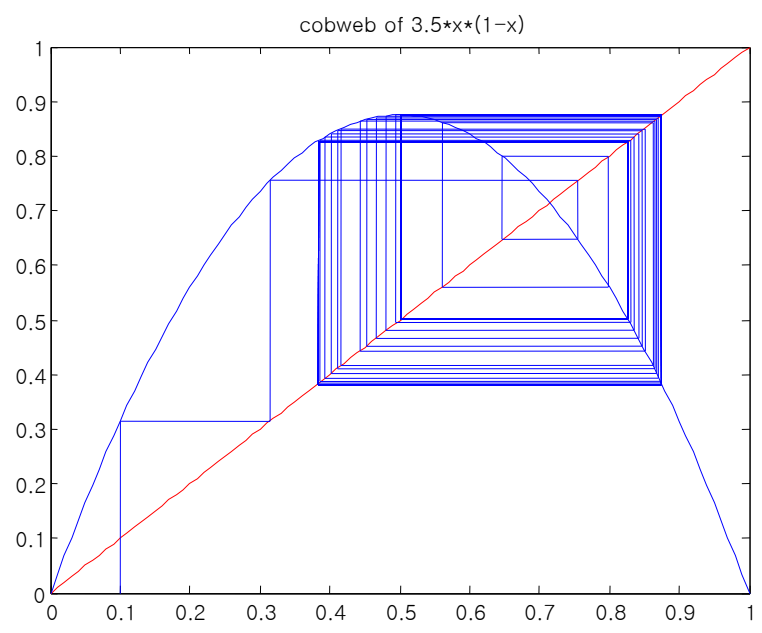

(b) Phase portrait and iteration with equilibrium point.

Figure 6. Time series and phase portrait with equilibrium point to addiction of digital leisure when $\mathrm{a}=3.5$.

\subsection{Bifurcation Diagram (Whole Stage)}

Using Eq. 6, we get no-addiction, pre-addiction, and addiction stages, corresponding to an equilibrium point, periodic doubling, and chaos, respectively, in Eq. (1).

Figure 8 shows a comparison between bifurcation diagrams and the addiction stage obtained from Eqs. (1) and (6), respectively. From the figure, we know that addiction to digital leisure occurs sequentially: no addiction, pre-addiction, and then addiction.

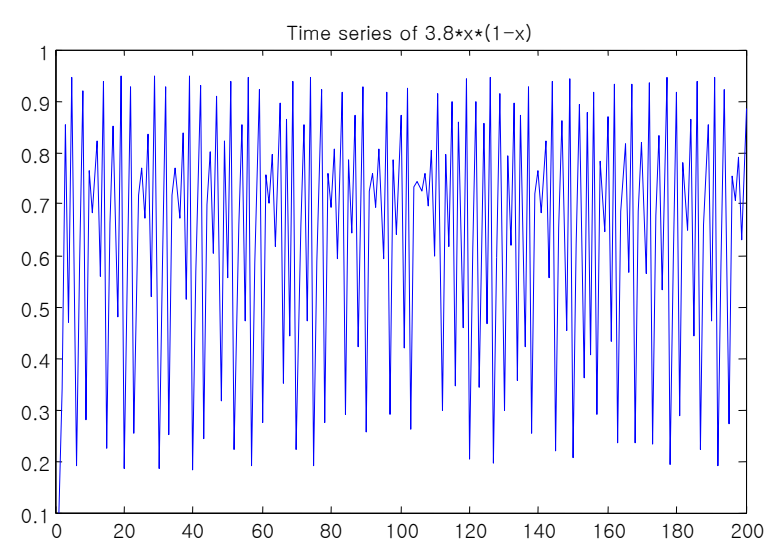

(a) Time series.

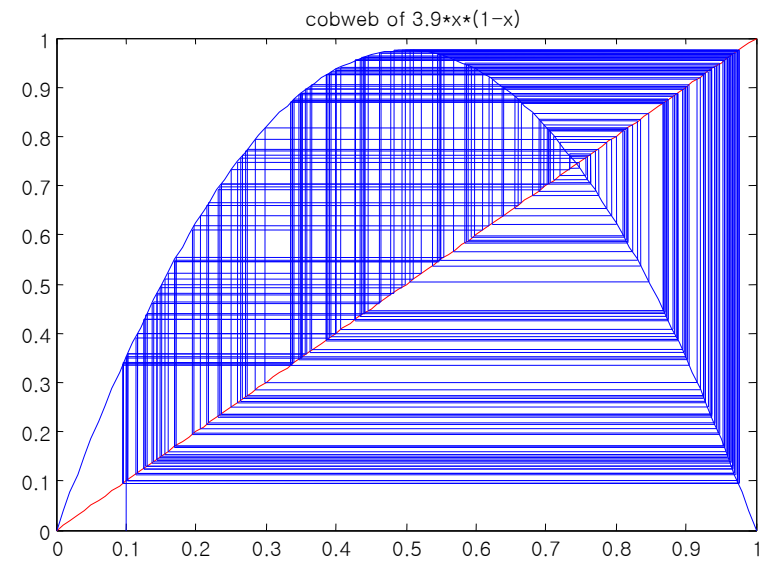

(b) Phase portrait and iteration with equilibrium point.

Figure 7. Time series and phase portrait with equilibrium point to addiction of digital leisure when $\mathrm{a}=3.9$.

\section{Conclusion}

We proposed a model of addiction to digital leisure such as the use of digital devices, including smart phones, game, and personal computers. To derive a mathematical model for addiction to digital leisure, we introduced a nicotine addiction model. Then, we substituted the nicotine addiction model into the logistic model. Finally, we subjected the logistic model to simulatione by varying the parameter $\gamma$. Consequently, we classified three stages of addiction: (i) a no-addiction stage, (ii) a pre-addiction stage, and (iii) an addiction stage. These three stages correspond to an equilibrium point, period-doubling, and chaos, respectively. 


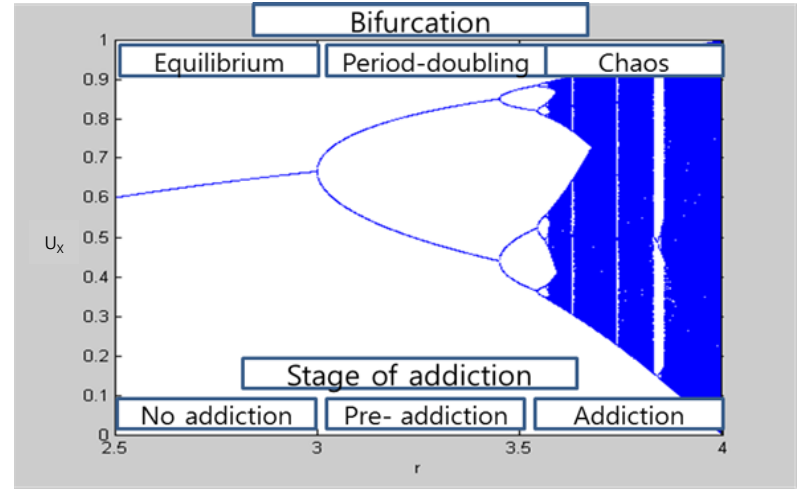

Figure 8. Bifurcation diagram of logistic map and stage of addiction to digital leisure.

\section{Acknowledgments}

This study was supported by the Ministry of Knowledge Economy (MKE) through the Regional Innovation Center Program.

\section{Conflict of Interest}

No potential conflict of interest relevant to this article was reported.

\section{References}

[1] A. Garfinkel, M. L. Spano, W. L. Ditto, and J. N. Weiss, "Controlling cardiac chaos," Science, vol. 257, no. 5074, pp. 1230-1235, Aug. 1992. http://dx.doi.org/10.1126/ science.1519060

[2] P. Faure and H. Korn, "Is there chaos in the brain? I. Concepts of nonlinear dynamics and methods of investigation," Comptes Rendus de l'Acadmie des Sciences Series III: Sciences de la Vie, vol. 324, no. 9, pp. 773-793, Sep. 2001. http://dx.doi.org/10.1016/S0764-4469(01)01377-4

[3] R. M. May, "Simple mathematical models with very complicated dynamics," Nature, vol. 261, no. 5560, pp. 459467, Jun. 1976. http://dx.doi.org/10.1038/261459a0

[4] Y. C. Bae, "A study on chaotic phenomenon in rolling mill bearing," Journal of Korean Institute of Intelligent Systems, vol. 11, no. 4, pp. 315-319, Aug. 2001.

[5] Y. C. Bae, J. W. Kim, Y. G. Kim, and Y. W. Shon, "Secure communication using embedding drive synchronization," Journal of Korean Institute of Intelligent Systems, vol. 13, no. 3, pp. 310-315, Jun. 2003. http://dx.doi.org/10.5391/ JKIIS.2003.13.3.310

[6] Y. C. Bae, Y. G. Kim, and M. Tinduka, "A study on generalized synchronization in the state-controlled cellular neural network (SC-CNN)," International Journal of Fuzzy Logic and Intelligent Systems, vol. 5, no. 4, pp. 291-296, Dec. 2005. http://dx.doi.org/10.5391/IJFIS.2005.5.4.291

[7] S. H. Yu, C. H. Hyun, and M. Park, "Backstepping control and synchronization for 4-D Lorenz-Stenflo chaotic system with single input," International Journal of Fuzzy Logic and Intelligent Systems, vol. 11, no. 3, pp. 143-148, Sep. 2011. http://dx.doi.org/10.5391/IJFIS.2011.11.3.143

[8] S. H. Yu, C. H. Hyun, and M. Park, "Control and synchronization of new hyperchaotic system using active backstepping design," International Journal of Fuzzy Logic and Intelligent Systems, vol. 11, no. 2, pp. 77-83, Jun. 2011. http://dx.doi.org/10.5391/IJFIS.2011.11.2.077

[9] Y. C. Bae, Y. G. Kim, and Y. D. Koo, "The target searching method in the chaotic mobile robot embedding BVP model," Journal of Korean Institute of Intelligent Systems, vol. 17, no. 2, pp. 259-264, Apr. 2007. http://dx.doi.org/10.5391/JKIIS.2007.17.2.259

[10] Y. C. Bae, Y. G. Kim, and C. S. Kim, "Obstacle avoidance method in the chaotic unmanned aerial vehicle," Journal of Korean Institute of Intelligent Systems, vol. 14, no. 7, pp. 883-888, Dec. 2004. http://dx.doi.org/10.5391/JKIIS. 2004.14.7.883

[11] Y. C. Bae, "Chaotic phenomena in MEMS with duffing equation," Journal of the Korea Institute of Electronic Communication Sciences, vol. 6, no. 5, pp. 709-716, Oct. 2011

[12] J. H. Kim, C. W. Park, E. T. Kim, and M. Park, "T-S fuzzy model-based adaptive synchronization of chaotic system with unknown parameters," Journal of Korean Institute of Intelligent Systems, vol. 15, no. 2, pp. 270-275, Apr. 2005. http://dx.doi.org/10.5391/JKIIS.2005.15.2.270

[13] E. S. Kim, "Dynamics of multi-dulayed feedback neural nets for the nonlinear capability and noise characteristics," Journal of Korean Institute of Intelligent Systems, vol. 9, no. 2, pp. 144-156, Apr. 1999. 
[14] H. C. Sung, J. B. Park, S. H. Go, and Y. H. Joo, "Intelligent digital redesign for uncertain nonlinear systems using power series," Journal of Korean Institute of Intelligent Systems, vol. 15, no. 7, pp. 881-886, Dec. 2005. http: //dx.doi.org/10.5391/JKIIS.2005.15.7.881

[15] Y. H. Choi and S. M. Kim, "Radial basis function network based predictive control of chaotic nonlinear systems," Journal of Korean Institute of Intelligent Systems, vol. 13, no. 5, pp. 606-613, Oct. 2003. http://dx.doi.org/10.5391/ JKIIS.2003.13.5.606

[16] S. Metin and N. S. Sengor, "Dynamical system approach in modeling addiction," in International Conference of Brain Inspired Cognitive Systems, Madrid, Spain, July 14-16, 2010.

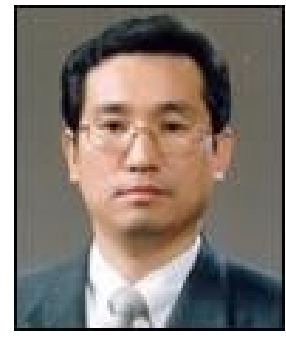

Youngchul Bae received his B.S., M.S., and Ph.D from the Department of Electrical Engineering, Kwangwoon University, Korea, in 1984,1985, and 1997, respectively. He worked at KEPCO (Korea Electric Power Company) during 1986-1991, and also worked at KISTI

(Korea Institute of Science and Technology Information) during 1991-1997 as a senior researcher. He is currently professor at the Division of Electrical, Electronic Communication and Computer Engineering, Chonnam National University, Yeosu, Korea. His research interests include nonlinear dynamics, chaos dynamics, robot control, intelligent system and motor control. He is member of KIEE, KIECS and KIIS.

E-mail: ycbae@chonnam.ac.kr 